This is an electronic reprint of the original article. This reprint may differ from the original in pagination and typographic detail.

Author(s): Baieva, Svitlana; Ihalainen, Janne; Toppari, Jussi

Title: $\quad$ Strong coupling between surface plasmon polaritons and \beta-carotene in nanolayered system

Year: $\quad 2013$

Version:

Please cite the original version:

Baieva, S., Ihalainen, J., \& Toppari, J. (2013). Strong coupling between surface plasmon polaritons and $\backslash$ beta-carotene in nanolayered system. Journal of Chemical Physics, 138(4), Article 044707. https://doi.org/10.1063/1.4776233

All material supplied via JYX is protected by copyright and other intellectual property rights, and duplication or sale of all or part of any of the repository collections is not permitted, except that material may be duplicated by you for your research use or educational purposes in electronic or print form. You must obtain permission for any other use. Electronic or print copies may not be offered, whether for sale or otherwise to anyone who is not an authorised user. 


\section{AlP The Journal of Chemical Physics}

\section{Strong coupling between surface plasmon polaritons and $\beta$-carotene in nanolayered system}

S. Baieva, J. A. Ihalainen, and J. J. Toppari

Citation: The Journal of Chemical Physics 138, 044707 (2013); doi: 10.1063/1.4776233

View online: http://dx.doi.org/10.1063/1.4776233

View Table of Contents: http://scitation.aip.org/content/aip/journal/jcp/138/4?ver=pdfcov

Published by the AIP Publishing

\section{Articles you may be interested in}

Temperature dependence of exciton-surface plasmon polariton coupling in $\mathrm{Ag}$, $\mathrm{Au}$, and $\mathrm{Al}$ films on InxGa1-xN/GaN quantum wells studied with time-resolved cathodoluminescence

J. Appl. Phys. 117, 043105 (2015); 10.1063/1.4906850

Strong coupling between surface plasmon polariton and laser dye rhodamine 800

Appl. Phys. Lett. 99, 051110 (2011); 10.1063/1.3619845

Enhancement of light transmission by coupling to surface plasmon polaritons of a layer-plus-islands silver layer J. Appl. Phys. 106, 103104 (2009); 10.1063/1.3259426

Surface plasmon polariton mediated photoluminescence from excitons in silicon nanocrystals Appl. Phys. Lett. 89, 101907 (2006); 10.1063/1.2345261

Bragg scattering of surface plasmon polaritons on extraordinary transmission through silver periodic perforated hole arrays

Appl. Phys. Lett. 88, 213112 (2006); 10.1063/1.2206553

\section{AIP $\left.\right|_{\text {APL Photonics }}$ \\ APL Photonics is pleased to announce Benjamin Eggleton as its Editor-in-Chief}

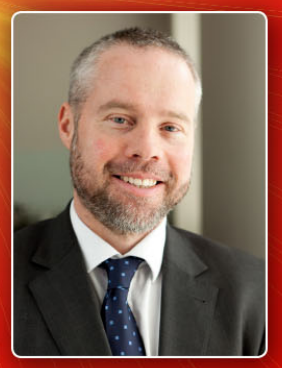




\title{
Strong coupling between surface plasmon polaritons and $\beta$-carotene in nanolayered system
}

\author{
S. Baieva, ${ }^{1}$ J. A. Ihalainen, ${ }^{2}$ and J. J. Toppari ${ }^{1}$ \\ ${ }^{1}$ Nanoscience Center, Department of Physics, University of Jyväskylä, Jyväskylä, \\ P.O. Box 35, FI-40014, Finland \\ ${ }^{2}$ Nanoscience Center, Department of Biological and Environmental Science, University of Jyväskylä, \\ Jyväskylä, P.O. Box 35, FI-40014, Finland
}

(Received 4 September 2012; accepted 2 January 2013; published online 24 January 2013)

\begin{abstract}
In this article we experimentally demonstrate the strong coupling between surface plasmon polaritons (SPP) and the $\mathrm{S}_{2}$ state of $\beta$-carotene. The SPPs are excited by prism coupling technique on a thin silver film with $\beta$-carotene embedded in a polymer layer on top of that. Rabi splittings with energies 80 and $130 \mathrm{meV}$ are observed in the recorded dispersion relations. Both coupled oscillator model and transfer matrix method are used to fit the experimental results. The scattered radiation of the propagating strongly coupled SPP-S $\mathrm{S}_{2}$ hybrids is collected and an increase of the low energy splitting to $120 \mathrm{meV}$ is observed compared to the reflectivity data. In addition, we performed molecule excitation by laser and analyzed the emission patterns revealing clear surface plasmon coupled fluorescence of $\beta$-carotene. By increasing the concentration of $\beta$-carotene we are able to collect also surface plasmon coupled Raman scattering. This study substantially extends the SPP-molecular excitation strong coupling studies to biomolecules, and energy transfer and coupling properties of excited states of carotenoids. (C) 2013 American Institute of Physics. [http://dx.doi.org/10.1063/1.4776233]
\end{abstract}

\section{INTRODUCTION}

In recent decades growing attention has been paid to experimental $^{1-9}$ and theoretical ${ }^{10}$ studies of surface plasmon polaritons (SPPs) strongly interacting with fluorescent molecules. SPP is a strongly coupled mode between light and coherently oscillating electrons on a metal surface, which can bypass the diffraction limit and still interact with optically active molecules similarly as photons. ${ }^{11-13}$ In the case of weak interaction, energy can be transferred from a SPP to a molecule, which then usually relaxes via fluorescence; or vice versa, the fluorescence near the metal can excite SPPs on it. ${ }^{14-18}$ This can be utilized on development of new nanodimensional photonic elements such as frequency converters, ${ }^{19}$ planar elements with desirable refractive index,${ }^{20}$ etc. Also, many modern spectroscopic techniques are based on the surface plasmons. For example, surface enhanced Raman spectroscopy (SERS) exploits electric field enhancement of plasmonic excitation that leads to an enormous increase in the Raman signal. ${ }^{21,22}$ Likewise, the surface plasmon coupled emission (SPCE) is a (bio)analytical tool that provides high fluorescence detection sensitivity and spatial separation of surface and bulk generated fluorescence. ${ }^{23-27}$ However, the high confinement of the SPP field and the large dipole moments of dye molecules can easily fulfill also the conditions for a strong coupling, where the molecule excited by SPPs does not anymore relax via fluorescence, but the energy is directly coupled back to the SPPs. In this regime the excitation becomes delocalized and SPP-molecule system must be considered as a whole, resulting in hybrid SPP-molecule states. ${ }^{5,8,28}$ The energy level splitting, also known as Rabi splitting, with the width directly related to the square root of the molecule concentration, is a signature of formation of such hybrid states. ${ }^{1-9}$
So far, to our knowledge, all experiments have been carried out by using high fluorescent dyes with a relatively simple energy state diagram. ${ }^{1-9,23-25}$ However, frequently in biosciences one has to deal with substances having a low fluorescence efficiency and a complicated excited state structure. One especially interesting optically active biomolecule is $\beta$-carotene, since among natural pigments the carotenoids have outstanding importance due to their functions in photosynthesis. ${ }^{29}$ In light-harvesting complexes, carotenoids are in close proximity to (bacterio)chlorophyll molecules resulting in a strong coupling between the excited states of the pigments. ${ }^{30}$ In recent years, it has become clear that the light induced excitation energy is transferred within and among the light-harvesting complexes via hybridized states of strongly coupled pigments. ${ }^{31-33}$ Thus, studies of strong coupling between the excited states of $\beta$-carotene and other type of excitation, like SPP, can reveal new information on energy transfer properties of carotenoids in natural systems as well. In addition, carotenoids are also potential candidates for molecular wires, ${ }^{34}$ and perturbing their conductivity by SPPs can reveal new aspects from it. ${ }^{35}$

In contrast to other dye molecules utilized in SPP studies, carotenoids show a strong electron correlation, a rather complicated excited state structure and short excited state lifetimes, and thus low fluorescence yield. Since the transition to the first excited state $S_{1}$ (with symmetry group of $2^{1} A_{g}$ ) is forbidden due to the molecule symmetry, the conventional absorption and fluorescence spectroscopy observes transitions involving second excited state of carotenoids, conventionally called $\mathrm{S}_{2}\left(1^{1} \mathrm{~B}_{\mathrm{u}}\right) \cdot{ }^{36}$ On the other hand, carotenoids have strong vibrational states resulting in intense resonance Raman signals. ${ }^{37}$ Since the vibrational levels play an essential role in 
the dynamics of the strongly coupled hybrid modes, ${ }^{38,39}$ the analysis of SPCE and scattered signals may reveal routes of mode relaxation via energy states that are not possibly approachable by any other spectroscopic techniques.

In this article, we report an extensive study of $\beta$-carotene $\mathrm{S}_{2}$ state strongly coupled to SPPs excited on a silver film. Within our knowledge this is the first study of coupling dynamics between SPPs and complex biologically important molecule.

\section{EXPERIMENTAL}

Samples were fabricated on top of a regular microscope glass slides with dimensions of $20 \mathrm{~mm} \times 20 \mathrm{~mm}$, and thickness of $1.5 \mathrm{~mm}$. The glasses were cleaned with hot acetone, followed by $5 \mathrm{~min}$ sonication in isopropyl alcohol, and drying by dry nitrogen flow. To remove organic residuals the reactive ion etching with oxygen plasma was used for $2 \mathrm{~min}$ with parameters: $50 \mathrm{SCCM}, 200 \mathrm{~W}$. After that, the silver layer was formed on top of the glass by electron-beam evaporation in an ultra-high $\left(10^{-8}\right.$ mbar $)$ vacuum. The characteristic thickness of the metal layer is $\sim 50 \mathrm{~nm}$, which was verified by an atomic force microscope. The evaporation rate of $0.02-0.05 \mathrm{~nm} / \mathrm{s}$ was chosen to obtain reasonably smooth surface (2.5 $\mathrm{nm}$ RMS).

SU-8 epoxy-based polymer negative resist (Microchem SU-8 2025) was used as a matrix for the molecules. One milligram of $\beta$-carotene (purchased from Fluka, no additional purification was done) was first dissolved in $1 \mathrm{ml}$ chloroform. After that the obtained solution was mixed with $10 \mu \mathrm{l}$ of SU-8 resist already diluted in cyclopentanone (1:6 volume ratio). Further filtering was used to remove possible aggregates with sizes above $0.2 \mu \mathrm{m}$. After the filtration the resist was spin-coated on top of the silver as shown in Fig. 1. To obtain the resist thickness of $\sim 40 \mathrm{~nm}$ the spinning rate of $4500 \mathrm{rpm}$ was used for $1 \mathrm{~min}$. Our typical routine of molecule doped resist handling requires 3 min sample baking at $95{ }^{\circ} \mathrm{C}$. In the case of $\beta$-carotene the heating was avoided,

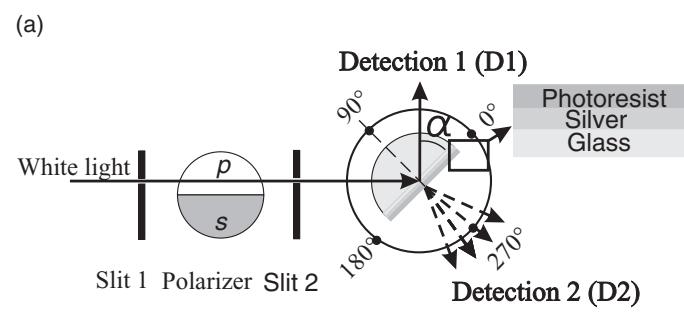

(b)

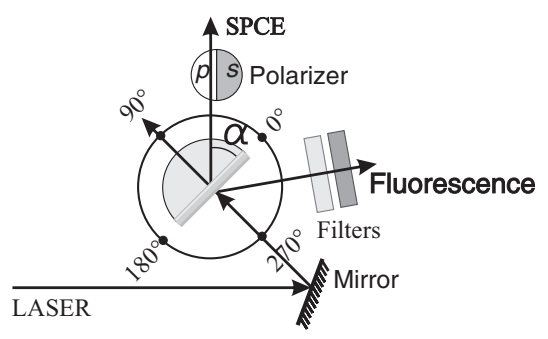

FIG. 1. Schematic representation of the experimental setup and the sample structure. $\alpha$ is the detection angle. because it led to extended $\beta$-carotene aggregation. For the absorption measurements reference samples without silver were similarly fabricated. The absorption spectra were measured by PerkinElmer UV/VIS Lambda 850 spectrometer at room temperature.

For each measurement set a new sample was always fabricated, since in a longer use the $\beta$-carotene layer may degrade due to the extensive light illumination and interaction with oxygen in air. ${ }^{40,41}$ Additionally, we had to consider an effect due to the unexposed SU-8 resin. The reference sample absorption changed more than two times after $1.5 \mathrm{~h}$ illumination with $488 \mathrm{~nm} \sim 90 \mu \mathrm{W} / \mathrm{mm}^{2}$ laser beam, whereas the sample without SU-8 did not change its absorption. Thus, in addition to have always a fresh sample for repetitive measurements, a special care was taken to minimize any effect of the film degradation.

Figure 1 shows schematics of our experimental setup. The hemicylindrical prism (ThorLabs) made of BK7 glass was used in Kretschmann configuration ${ }^{12,13}$ and a sample was installed on the flat face of the prism by index matching oil. Oriel 66182 white light source was used for excitation (Fig. 1(a)). The light was collimated and aligned by two slits with a rotatable prism polarizer in between to adjust the polarization. The incident angle of the incoming light was adjusted manually by rotating the goniometric prism mount. The reflected, detection 1 (D1), and scattered (D2) signals were collected by a collimating optics assembly F220SMA-A (ThorLabs) $\mathrm{f}=10.9 \mathrm{~mm}, \mathrm{NA}=0.25$ that was connected to an optic fiber. The radiation was guided to Jobin Yvon iHR320 spectrometer equipped with Jobin Yvon Symphony CCD camera. It should be noted that the reflected and the scattered signals were not collected simultaneously.

To perform the second part of the study, the setup was slightly modified to the configuration shown in Fig. 1(b). The sample with $\beta$-carotene was excited from the molecule side with $488 \mathrm{~nm}$ or $514 \mathrm{~nm}\left(\sim 70 \mu \mathrm{W} / \mathrm{mm}^{2}\right)$ lines from Stelar-Pro multi-line argon laser (Modu-Lasers), and the emission was collected from the prism side as well as from the molecule side. This method of excitation is often referred as a reverse Kretschmann configuration. ${ }^{22}$

To collect the emission from the $270^{\circ}-360^{\circ}$ region, i.e., from the molecule side, the detection unit was equipped with stained glass filters to cut the excitation radiation. In the prism side, i.e., angles $0^{\circ}-90^{\circ}$, the detection filtering is not required, since the amount of scattered radiation from the transmitted beam is reduced by focusing properties of the prism, and the region of the emission, i.e., $33^{\circ}-42^{\circ}$, is far from the transmitted beam. To check the polarization of the emission the polarizer was introduced into the detection line in both regions.

\section{RESULTS AND DISCUSSION}

Examples of the measured absorption spectra are shown in Fig. 2. For the further modeling, the absorption spectrum of the $\beta$-carotene film was fitted with four Lorentzian curves associated with four vibrational bands of the $S_{0} \rightarrow S_{2}$ transition. The peaks are located at 508 (0-0 transition), 472 (0-1), 443 (0-2), and $421 \mathrm{~nm}(0-3)$. The overall redshift of the thin film spectra as compared to the highly diluted solution spectrum 


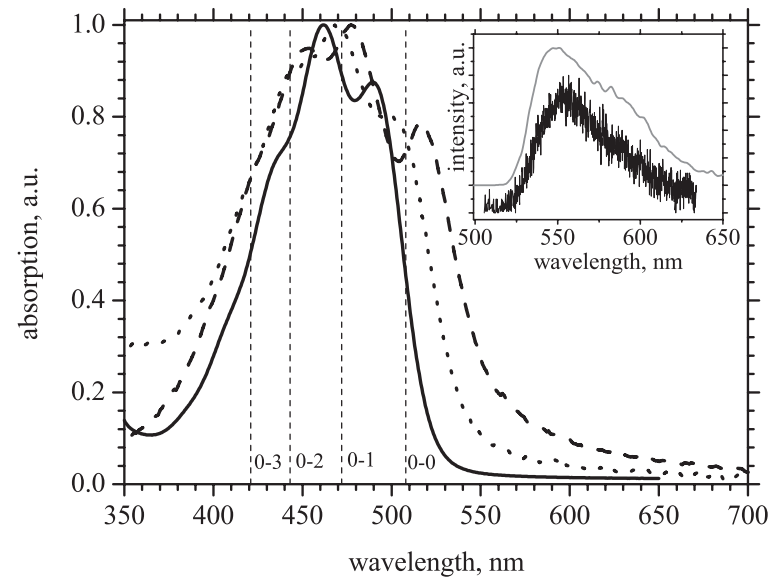

FIG. 2. Absorption spectra of $\beta$-carotene monomer solution in chloroform, as well as of SU-8 thin films fabricated from solutions containing $1 \mathrm{mg}$ and $3 \mathrm{mg}$ of $\beta$-carotene are plotted as solid, dotted and dashed lines, respectively. The spectra are normalized to their maxima. The vertical dashed lines indicate the $\mathrm{S}_{2}$ vibrational transitions of the $1 \mathrm{mg}$ film. The redshift of the bands compared to the diluted solution increases with increasing $\beta$-carotene concentration. The inset shows the fluorescence spectra of $\beta$-carotene measured from the $1 \mathrm{mg}$ film (black curve) and from the diluted solution (gray curve) using $488 \mathrm{~nm}$ excitation. Vertical offset is added for clarity.

with the bands at $494,461,433$, and $411 \mathrm{~nm}$ is most likely due to a J-aggregate formation. The tendency of formation of $\mathrm{H}$ - and J-aggregates in hydrated polar solvents is well-known for carotenoids, ${ }^{42,43}$ and is controlled by solvent properties and molecule concentration. In the present case the high concentration of $\beta$-carotene in the initial solution leads to a presence of some J-aggregates in the film. Subsequent drying of the film has minor effect on the aggregate formation and therefore on the absorption spectra. Also, the fluorescence of the $\beta$-carotene film on top of a silver layer was recorded at angles $270^{\circ}-360^{\circ}$ by the setup shown in Fig. 1(b). The obtained fluorescence spectra agreed very well with the spectrum recorded from a $\beta$-carotene solution as shown in the inset of Fig. 2.

The typical data obtained via Kretschmann configuration by D1 and D2 are shown in Fig. 3(a). The reflected light spectrum (D1) was collected for every angle of incidence higher than the total internal reflection angle and divided by the spectrum collected at $0^{\circ}$ angle of incidence bypassing the prism and the sample. Finally, the data were normalized to the highest value over the whole range. The scattered radiation data were collected at D2 with the same angles, but only normalized to the highest value over the whole range. The coupling to SPPs manifests itself as minima in the reflected spectra and maxima in scattered radiation spectra.

We analyzed the experimental data using two approaches-transfer matrix method ${ }^{44}$ and coupled oscillators model. ${ }^{45}$ First one is based on Fresnel equations for multilayered structures. The model system used was multilayered structure that consists of semi-infinite dispersionless glass (dielectric constant $\varepsilon_{g}=2.43$ ), $50 \mathrm{~nm}$ silver layer with dielectric function calculated using the data from Ref. 46, $30 \mathrm{~nm}$ of resist containing $\beta$-carotene, and semi-infinite air layer. The dielectric function of the $\beta$-carotene layer can be (a)

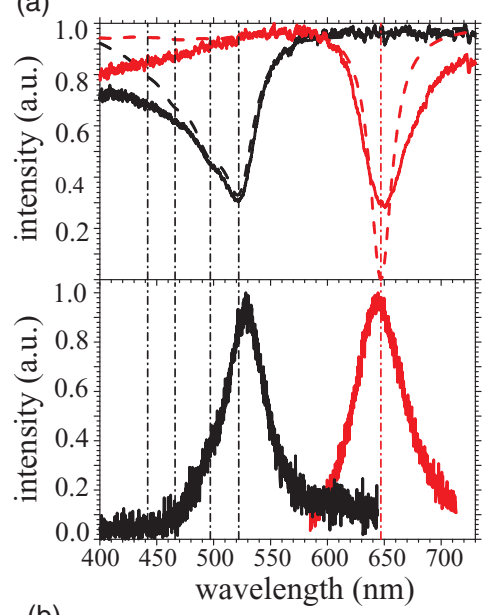

(b)

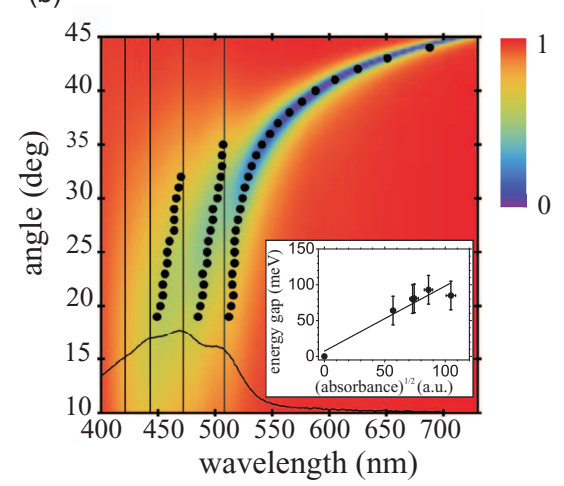

FIG. 3. (a) Measured reflectance, D1 (upper curves) and scattered radiation intensity (lower curves) as a function of the excitation wavelength at the $30^{\circ}$ (black line) and $43^{\circ}$ (red line) excitation angles. Dashed lines represent calculated reflectance spectra at the corresponding angles of incidence. Vertical dashed-dotted lines indicate positions of the fitted intensity minima on the upper image. (b) Calculated reflectance coefficient as a function of the excitation wavelength and angle (color scale) together with the measured minima of the reflected light spectra, D1 (black circles). Black curve is the measured absorption spectrum of the same $\beta$-carotene thin film, and the vertical black lines indicate positions of its maxima. The inset represents the dependence of the width of the lowest $(0-0)$ energy gap on a square root of the total absorbance of the $\beta$-carotene film together with its linear fit.

described by an equation

$$
\varepsilon(\omega)=\varepsilon_{S}+\sum_{i=1}^{4} \frac{A_{i} \omega_{0 i}^{2}}{\omega_{0 i}^{2}-\omega^{2}-i \omega \gamma_{i}},
$$

where $\varepsilon_{s}=3$ is a frequency independent dielectric permittivity of the media that is hosting the $\beta$-carotene molecule, i.e., SU-8 resist in our case. $A=\{0.0200,0.0260,0.0236,0.0130\}$ is a dimensionless parameter characterizing the strength of an oscillation with the corresponding resonance frequency $\omega_{0}=2 \pi / \lambda=\{1236.47,1330.41,1416.90,1493.52\}$ $\times 10^{4} \mathrm{~m}^{-1}$, where $\lambda$ is the wavelength, and $\gamma=\{0.91,1.20$, $1.60,1.60\} \times 10^{8} \mathrm{~m}^{-1}$ describes damping of such oscillation. We deduced parameters $A_{i}, \omega_{0 i}$, and $\gamma_{i}$ from the measured absorption spectra of the reference samples containing only the $\beta$-carotene resist film spun on the glass substrate. Figure 3(b) shows calculated reflectance coefficient of the modeled system together with the experimental data showing the positions of the minima obtained by fitting Lorentzian curves to the dips in the reflectance, i.e., D1. Both data clearly 
show opening of the energy gaps at the transitions, and thus, the formation of the strongly coupled SPP-S $\mathrm{S}_{2}$ hybrid modes. It is important to realize that D2 data result from SPPs scattering on silver film imperfections and it is not the transmittance in terms of the transfer matrix method. D2 signal is also affected by the SPP- $\beta$-carotene interaction that is beyond the model described above. So, the D2 data cannot be fitted with the transfer matrix method directly.

We are able to reliable resolve three hybrid branches in D1 geometry, i.e., energy splittings corresponding to the strong coupling between SPP and the $\beta$-carotene transitions $(0-0)$ and (0-1), and two branches in D2, i.e., only the splitting with (0-0) transition was resolvable due to the reduced amplitudes of the higher energy modes. We call them 0-0 and $0-1$ splits in the further discussion. The higher energy branches in D1 (and D2) cannot be resolved due to their increasing linewidths and reduced amplitudes as also visible in the calculated transfer matrix data in Fig. 3(b). The excess decrease of the intensity of the higher energy branches in the scattered radiation, D2, compared to D1, has been observed also earlier. ${ }^{3,47}$ There are several possible reasons for that; higher Ohmic losses for higher energies of SPPs, energy transfer between the branches or between the SPP-S hybrid states and uncoupled $\mathrm{S}_{2}$-excitations, ${ }^{34}$ relaxation to vibrational reservoir ${ }^{38,39}$ are among them.

The dispersion curves, i.e., energy dependence of the $\mathrm{SPP}-\mathrm{S}_{2}$ mode on the in-plane wavevector component $k_{x}$, can be deduced from the fitted extrema of D1 or D2. Experimental dispersion relations together with the fits by the coupled oscillators model ${ }^{1,3,47}$ are shown in Fig. 4. The energy gaps are clearly visible at the positions of the two lowest $\beta$-carotene film absorption maxima, i.e., (0-0) and (0-1). In D1 the $0-0$ and $0-1$ splits are $80 \pm 20$ and $130 \pm 20 \mathrm{meV}$, correspondingly, while the D2 data yield $120 \pm 20 \mathrm{meV}$ for the 0-0 gap. These values were derived from the fitted coupling strength parameters in a coupled oscillator model calculation. ${ }^{45}$ The change of the energy gap value between

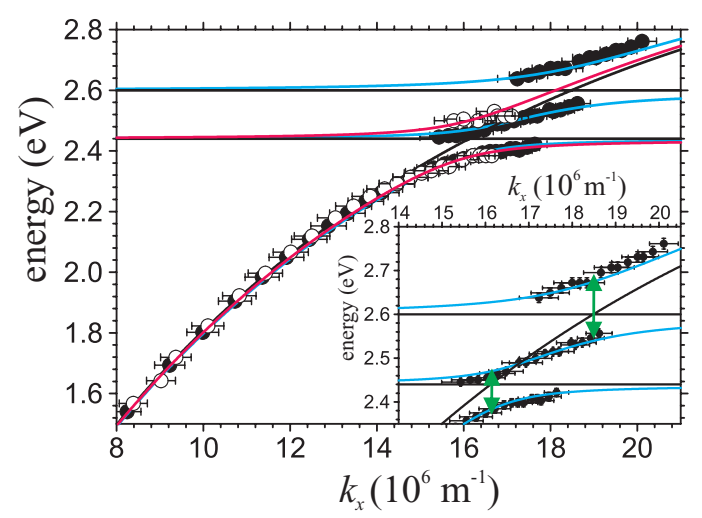

FIG. 4. (a) The dispersion curves obtained in D1 (black circles) and D2 (empty circles). Black horizontal lines represent the two lowest absorption maxima of $\beta$-carotene, i.e., (0-0) and (0-1); black curve is the SPP dispersion relation of a sample without $\beta$-carotene. Blue and pink curves are results from the coupled oscillator model fitted to the D1 and D2 data, respectively. Inset shows the zoomed gap regions of the D1 dispersion relation. Crossings between the dispersion relation of a sample without $\beta$-carotene and $\beta$-carotene absorption maxima and the widths of the opened splittings are indicated as arrows.
D1 and D2 is explained by increased interaction time between SPP and molecules. We refer to earlier papers for the detailed explanation of the phenomenon. ${ }^{3,47}$ Also a concentration study was done for the width of the lowest (0-0) split, and as expected in the case of strong coupling, the split width was linearly dependent on the square root of the total absorption as shown in the inset of Fig. 3(b). The change of the excitation intensity, more than an order of magnitude, did not affect the dispersion at all, implying the energy gaps being vacuum Rabi splits.

In order to understand the radiation patterns observed in the reverse Kretschmann configuration experiments we refer to models of dipole radiation near the interface. The models are dealing with application of Fresnel coefficients to the dipole $^{48}$ or multipole ${ }^{49}$ fields. We considered a multilayer system that includes semi-infinite air, $30 \mathrm{~nm}$ dielectric layer with $\beta$-carotene dielectric function (Eq. (1)); $50 \mathrm{~nm}$ silver layer with dielectric function calculated using data from Ref. 46, and semi-infinite glass medium. Dipoles are positioned at the boundary between the air and the dielectric medium. Also, it is assumed that the orientation of the dipoles is random and they radiate uniformly in energy domain. We present calculation of the $p$-polarized radiation profile together with the measured one for $570 \mathrm{~nm}$ wavelength in Fig. 5(a). The measurements were done with excitation by 488 and $514 \mathrm{~nm}$ laser and it agrees very well with the calculated profile. In $0^{\circ}-90^{\circ}$ region we observe strongly directed emission at $38^{\circ}$ that is SPCE. Due to the SPP dispersion relation the certain molecule coupled transition is capable to excite SPP with a unique wavevector, so the angular position of SPCE is dependent on the radiation energy. As a result also the measured spectrum is dependent on the detection angle as seen in Figs. 5(b) and $5(\mathrm{c})$. Radiation in $270^{\circ}-360^{\circ}$ region, i.e., on the molecular side, is the $\beta$-carotene fluorescence (Fig. 2 inset). Its intensity distribution is Lambertian and the spectrum is the same at every angle.

Collecting of SPCE from the prism side is another way to obtain the part of the dispersion relation. We used 488 and $514 \mathrm{~nm}$ lasers for the excitation, so we were able to obtain the emission from the lower energy states of the $\beta$-carotene that were further coupled to SPPs or the SPP-S $S_{2}$ hybrid states. Yet, as the fluorescence spectrum of the $\beta$-carotene extends from 520 to $620 \mathrm{~nm}$ (see Fig. 2), only the lowest energy branch of the dispersion relation was accessible as shown in Fig. 6. It should be stressed that in our case the term SPCE is loosely used since the fluorescence is most probably coupled to the SPP-S $\mathrm{S}_{2}$ hybrid modes rather than to pure SPPs. Since at the region where the SPCE was observed, the hybrid mode is almost purely plasmonic, we cannot confidently deduce whether the emission is mediated via pure SPPs or the hybrids. In any case, the overlap between the D1 data and SPCE dispersion curve proves the radiation being mediated by SPPs or the hybrid states, as seen on the inset in Fig. 6. This was especially clear while using the $488 \mathrm{~nm}$ excitation (Fig. 6(a)). When $514 \mathrm{~nm}$ excitation wavelength was used a weak extra angle independent peak appeared at $557 \mathrm{~nm}$ in the SPCE spectra as shown in Fig. 5(c). To investigate the nature of the peak, we fabricated and measured a sample with higher $\beta$-carotene concentration. The $3 \mathrm{mg} \beta$-carotene (instead of $1 \mathrm{mg}$, as so 
(a)

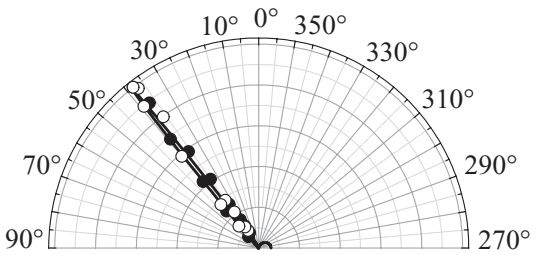

(b)

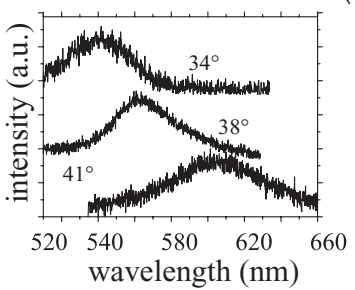

(c)

(d)
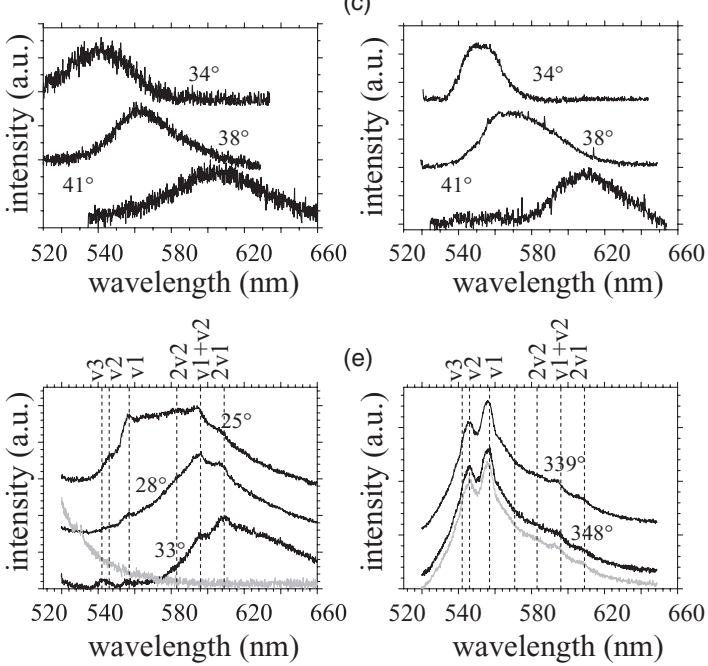

FIG. 5. (a) Calculated radiation profile (see details in text) plotted in polar coordinates (black curve) together with the measured radiation profiles for $570 \mathrm{~nm}$ excited with 488 and $514 \mathrm{~nm}$ (empty and black circles, correspondingly). (b) and (c) Measured SPCE of the $1 \mathrm{mg} \beta$-carotene sample at several detection angles. Excitation wavelengths were 488 (b) and $514 \mathrm{~nm}$ (c). (d) Measured $p$-polarized SPP coupled Raman signals of the $3 \mathrm{mg} \beta$ carotene sample at several detection angles (black curves) and corresponding $s$-polarized signal (gray curve) with $514 \mathrm{~nm}$ excitation. (e) Measured $p$ polarized (black curves) and $s$-polarized (gray curve) Raman spectra of the $3 \mathrm{mg} \beta$-carotene sample at several detection angles from the molecular side with $514 \mathrm{~nm}$ excitation. The dashed lines in (d) and (e) indicate positions of the Raman shifted bands. In all the images the spectra have been offset for clarity.

far) were dissolved and spin-coated according to the procedure described earlier in Sec. II. The measurement results of this high concentration sample are shown in Figs. 5(d), 5(e), and $6(\mathrm{~b})$, in which one can clearly see extra peaks as angle independent lines. We assigned those peaks to $\beta$-carotene Raman active vibrations $\mathrm{v}_{1}=1525 \mathrm{~cm}^{-1}, \mathrm{v}_{2}=1155 \mathrm{~cm}^{-1}$, $\mathrm{v}_{3}=1005 \mathrm{~cm}^{-1}$ and their combinations $2 \mathrm{v}_{1}, 2 \mathrm{v}_{2}$, and $\mathrm{v}_{1}+\mathrm{v}_{2}$ according to Ref. 37.

The detector was equipped with polarizer and the sample emission polarization was also tested with all the angles. Figure 5(e) shows the presence of the same sharp Raman peaks in $270^{\circ}-360^{\circ}$ region regardless of the detected polarization, while only $p$-polarized peaks were observed in $0^{\circ}-90^{\circ}$ region as shown in Fig. 5(d). The absence of the $s$-polarized signal in the $0^{\circ}-90^{\circ}$ region and the intensity dependence of the peaks on the detection angle proves that the Raman scattered radiation was coupled to SPPs or SPP-S 2 hybrid modes and mediated by them, and not appearing at the prism side due to interaction with leaking laser radiation. It is also interesting to note that the observed Raman shifts values are exactly the same as those reported in Ref. 37 for $\beta$-carotene solution in isopentane, while the absorption spectrum reported by authors shows no signs of J-aggregates, but is similar to our spectrum
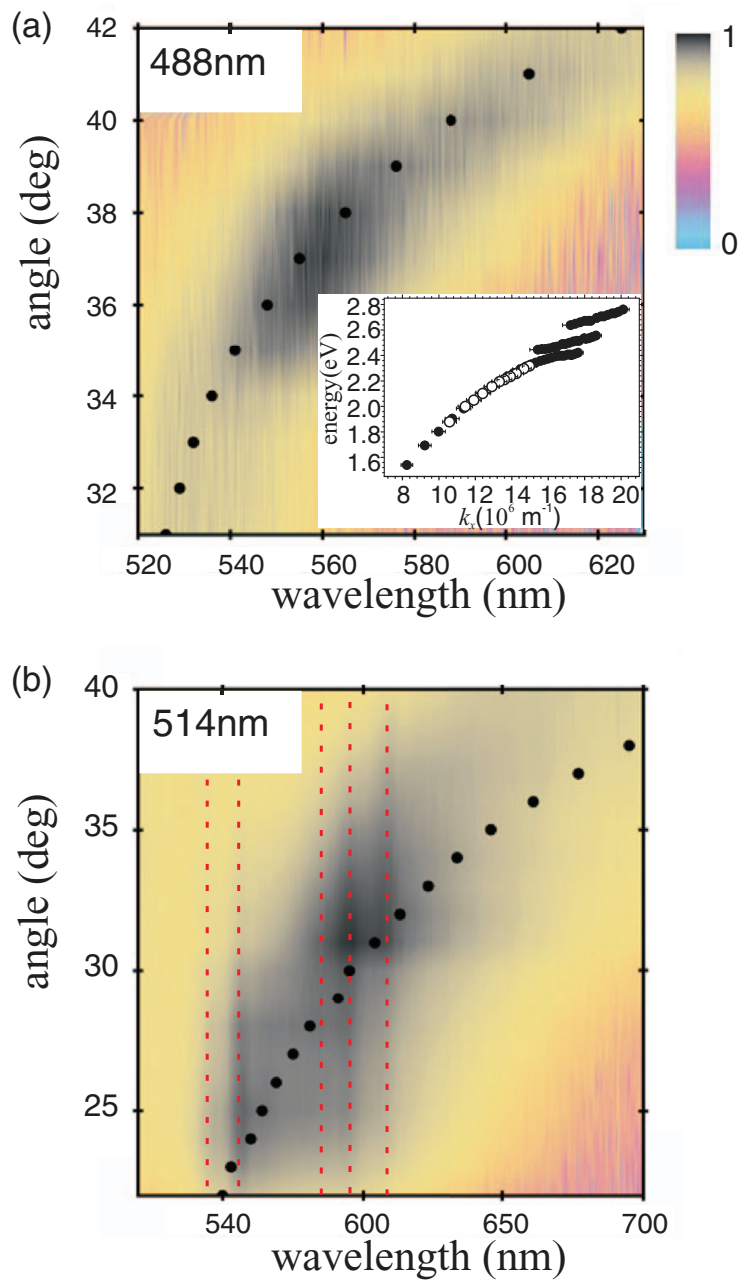

FIG. 6. SPCE from the $1 \mathrm{mg}$ sample excited with 488 (a) and from the $3 \mathrm{mg}$ sample excited with $514 \mathrm{~nm}$ (b) as a function of the wavelength and the detection angle together with the measured minima of the reflected light spectra (black circles). Inset on plot (a) shows the dispersion relations deduced from the reflectance measurement (black dots) and SPCE (white dots). The thin vertical lines work as a guide for eye for the observed Raman bands. The color scale is presented in a logarithmic scale for clarity.

of diluted $\beta$-carotene solution in chloroform presented above (Fig. 2).

Observation of Raman scattering between two SPP channels has been reported for n-hexane, ${ }^{50}$ and also for strongly coupled microcavity modes in Ref. 51 for a planar microcavity containing J-aggregates of a cyanine dye. In both of these experiments the initial excitation was done by SPP or strongly coupled cavity photons, which further scattered by molecule vibrations to another SPP or cavity photon states, and the consequent scattering of them into photons was detected. Since in our case the excitation was done by far field laser from the molecules side, we adapt a mechanism similar to the SPCE process to interpret our data. The initial far field excitation is directly exciting the molecule (resonantly or virtually) and the subsequent emission to another vibrational state inducing the Raman shift, is coupled to SPPs via its near-field. Then SPP travels along the metal surface before scatters to photons that are detected. The presence of both the $s$ - and $p$-polarized Raman bands in $180^{\circ}-270^{\circ}$ region strongly supports this model, 
since to obtain the $s$-polarized radiation, part of the radiation have to be direct Raman scattering without coupling to SPPs on the way.

In the case of $\beta$-carotene excited by the $488 \mathrm{~nm}$ laser, the absence of the SPP mediated Raman peaks is predictable. If one assumes the Raman shifts mentioned above, the corresponding peaks would appear in the gap energies or close to the gap region. So, a system in the strong coupling regime does not maintain those modes, and one only observes SPCE. In addition, according to resonance Raman scattering spectra reported in Ref. 52 for all-trans- $\beta$-carotene crystals the $1525 \mathrm{~cm}^{-1}$ is much weaker for excitation with $488 \mathrm{~nm}$. On the contrary, by using the excitation of $514 \mathrm{~nm}$ the Raman peaks fall within the range of the supported modes. In the case of the fluorescence, the non-radiative relaxation rate seems to increase with the concentration of the $\beta$-carotene, whereas the Raman efficiency remains constant. Thus, in the high concentration sample the fluorescence of $\beta$-carotene is quenched due to extra non-radiative decay routes provided by the increased amount of J-aggregates, which explains the enhanced Raman peaks observed. We did not observe clear Raman peaks with 1 $\mathrm{mg} \beta$-carotene sample even with $514 \mathrm{~nm}$ excitation as shown in Fig. 5(b). So, we effectively transformed the emission from the SPCE to the SPP coupled Raman by increasing the $\beta$ carotene concentration. The corrugations of silver layer can also cause the enhancement of Raman signals. We think that the effect of the silver film roughness was small since the films were fabricated in the same conditions and were continuous and smooth.

\section{CONCLUSIONS}

We have detected strong coupling of SPPs to the two lowest vibrational bands of the $\mathrm{S}_{2}$ state of the $\beta$-carotene. We show that albeit of the complex excited state dynamics and low emission yield, the $S_{2}$ excited states are strongly coupled to SPPs with Rabi splittings and coupling energies up to $130 \mathrm{meV}$. On the other hand, we did not observe any coupling to the forbidden $S_{1}$ state of the carotenes, basically due to its very low transition dipole moment. Also a strong surface plasmon coupled emission, together with a surface enhanced and SPP coupled Raman signal were detected. The Raman signal was found to get more dominant over the fluorescence with increased $\beta$-carotene concentration. These results share light to many interesting phenomena of strong coupling between biologically important molecules and optical excitations.

\section{ACKNOWLEDGMENTS}

The authors thank Heli Lehtivuori and Päivi Ruokola for help in the supporting fluorescence and Raman measurements, Juha Linnanto for discussions of properties of $\beta$-carotene, Mikko Koponen for help in measurements and fabrication of samples, Pasi Myllyperkiö for help in optical set up, Thomás Polívka for providing information about the aggregation properties of $\beta$-carotenes. For financial support the authors thank Academy of Finland (Project Nos. 135193, 218182, and 134061), EU's COST action MP0803: Plasmonic
Components and Devices, and National Doctoral Programme in Nanoscience.

${ }^{1}$ J. Bellessa, C. Bonnand, J. C. Plenet, and J. Mugnier, Phys. Rev. Lett. 93, 036404 (2004).

${ }^{2}$ J. Dintinger, S. Klein, F. Bustos, W. L. Barnes, and T. W. Ebbesen, Phys. Rev. B 71, 035424 (2005).

${ }^{3}$ T. K. Hakala, J. J. Toppari, M. Pettersson, A. Kuzyk, H. Tikkanen, H. Kunttu, and P. Törmä, Phys. Rev. Lett. 103, 053602 (2009).

${ }^{4}$ G. P. Wiederrecht, J. E. Hall, and A. Bouhelier, Phys. Rev. Lett. 98, 083001 (2007).

${ }^{5}$ A. Salomon, C. Genet, and T. W. Ebbesen, Angew. Chem., Int. Ed. 48, 8748 (2009).

${ }^{6}$ F. Valmorra, M. Bröll, S. Schwaiger, N. Welzel, D. Heitmann, and S. Mendacha, Appl. Phys. Lett. 99, 051110 (2011).

${ }^{7}$ T. Schwartz, J. A. Hutchison, C. Genet, and T. W. Ebbesen, Phys. Rev. Lett. 106, 196405 (2011).

${ }^{8}$ A. Salomon, R. J. Gordon, Y. Prior, T. Seideman, and M. Sukharev, Phys. Rev. Lett. 109, 073002 (2012).

${ }^{9}$ S. Aberra Guebrou, C. Symonds, E. Homeyer, J. C. Plenet, Yu. N. Gartstein, V. M. Agranovich, and J. Bellessa, Phys. Rev. Lett. 108, 066401 (2012).

${ }^{10}$ D. E. Chang, A. S. Sørensen, P. R. Hemmer, and M. D. Lukin, Phys. Rev. B 76, 035420 (2007).

${ }^{11}$ H. A. Atwater, Sci. Am. 296, 56 (2007).

${ }^{12}$ S. A. Maier, Plasmonics: Fundamentals and Applications (Springer, New York, 2006).

${ }^{13}$ A. V. Zayats, I. I. Smolyaninov, and A. A. Maradudin, Phys. Rep. 408, 131 (2005).

${ }^{14}$ A. Archambault, F. Marquier, J. J. Greffet, and C. Arnold, Phys. Rev. B 82, 035411 (2010).

${ }^{15}$ W. H. Weber and C. F. Eagen, Opt. Lett. 4, 236 (1979).

${ }^{16}$ W. L. Barnes, J. Mod. Opt. 45, 661 (1998).

${ }^{17}$ P. Andrew and W. L. Barnes, Science 306, 1002 (2004).

${ }^{18}$ H. Ditlbacher, J. R. Krenn, N. Felidj, B. Lamprecht, G. Schider, M. Salerno, A. Leitner, and F. R. Aussenegg, Appl. Phys. Lett. 80, 404 (2002).

${ }^{19}$ T. K. Hakala, J. J. Toppari, M. Pettersson, A. Kuzyk, H. Tikkanen, H. Kunttu, and P. Törmä, Appl. Phys. Lett. 93, 123307 (2008).

${ }^{20}$ J. A. Hutchison, D. M. O'Carroll, T. Schwartz, C. Genet, and T. W. Ebbesen, Angew. Chem., Int. Ed. 50, 2085 (2011).

${ }^{21}$ P. L. Stiles, J. A. Dieringer, N. C. Shah, and R. P. Van Duyne, Annu. Rev. Anal. Chem. 1, 601 (2008).

${ }^{22}$ M. Moskovits and J. S. Suh, J. Phys. Chem. 88, 5526 (1984).

${ }^{23}$ J. R. Lakowicz, I. Gryczynski, J. Malicka, and Z. Gryczynski, Biochem. Biophys. Res. Commun. 307, 435 (2003).

${ }^{24}$ J. R. Lakowicz, Anal. Biochem. 324, 153 (2004).

${ }^{25}$ I. Gryczynsk, J. Malicka, Z. Gryczynski, and J. R. Lakowicz, Anal. Biochem. 324, 170 (2004)

${ }^{26}$ J. Enderlein and T. Ruckstuhl, Opt. Express 13, 8855 (2005).

${ }^{27}$ T. Ruckstuhl, M. Rankl, and S. Seeger, Biosens. Bioelectron. 18, 1193 (2003).

${ }^{28}$ N. T. Fofang, N. K. Grady, Z. Fan, A. O. Govorov, and N. J. Halas, Nano Lett. 11, 1556 (2011).

${ }^{29}$ T. Polívka and V. Sundström, Chem. Rev. 104, 2021 (2004).

${ }^{30}$ B. P. Krueger, G. D. Scholes, and G. R. Fleming, J. Phys. Chem. B 102, 5378 (1998).

${ }^{31}$ R. van Grondelle and V. I. Novoderezhkin, Phys. Chem. Chem. Phys. 8, 793 (2006).

${ }^{32}$ J. M. Linnanto and J. E. I. Korppi-Tommola, Chem. Phys. 357, 171 (2009).

${ }^{33}$ G. S. Engel, T. R. Calhoun, E. L. Read, T.-K. Ahn, T. Mančal, Y.-C. Cheng, R. E. Blankenship, and G. R. Fleming, Nature (London) 446, 782 (2007).

${ }^{34}$ G. K. Ramachandran, J. K. Tomfohr, J. Li, O. F. Sankey, X. Zarate, A. Primak, Y. Terazon, T. A. Moore, A. L. Moore, D. Gust, L. A. Nagahara, and S. M. Lindsay, J. Phys. Chem. B 107, 6162 (2003).

${ }^{35}$ T. Isoniemi, A. Johansson, T. K. Hakala, M. Rinkiö, P. Törmä, J. J. Toppari, and H. Kunttu, Appl. Phys. Lett. 99, 031105 (2011).

${ }^{36}$ A. N. Macpherson and T. Gillbro, J. Phys. Chem. A 102, 5049 (1998).

${ }^{37}$ Z. Ho, R. C. Hanson, and S. H. Lin, J. Chem. Phys. 77, 3414 (1982).

${ }^{38}$ D. M. Coles, P. Michetti, C. Clark, A. M. Adawi, and D. G. Lidzey, Phys. Rev. B 84, 205214 (2011).

${ }^{39}$ D. M. Coles, P. Michetti, C. Clark, W. C. Tsoi, A. M. Adawi, J. S Kim, and D. G. Lidzey, Adv. Funct. Mater. 21, 3691 (2011).

${ }^{40}$ S. Limbo, L. Torri, and L. Piergiovanni, J. Agric. Food Chem. 55, 5238 (2007). 
${ }^{41}$ L. K. Henry, N. L. Puspitasari-Nienaber, M. Jarén-Galán, R. B. Breeme, G. L. Catignani, and S. J. Schwartz, J. Agric. Food Chem. 48, 5008 (2000).

${ }^{42}$ H. H. Billsten, V. Sundström, and T. Polívka, J. Phys. Chem. A 109, 1521 (2005).

${ }^{43}$ F. Zsila, Z. Bikádi, J. Delib, and M. Simonyia, Tetrahedron. Lett. 42, 2561 (2001).

${ }^{44}$ B. Harbecke, Appl. Phys. B 39, 165 (1986).

${ }^{45}$ R. J. Holmes and S. R. Forrest, Phys. Rev. Lett. 93, 186404 (2004).

${ }^{46}$ R. W. Christy and P. B. Johnson, Phys. Rev. B 6, 4370 (1972).
${ }^{47}$ S. V. Baieva, T. K. Hakala, and J. J. Toppari, Nanoscale Res. Lett. 7, 191 (2012).

${ }^{48}$ L. Novotny and B. Hecht, Principles of Nano-Optics (Cambridge University Press, New York, 2006), p. 335.

${ }^{49}$ H. F. Arnoldus, J. Opt. Soc. Am. 22, 190 (2005).

${ }^{50}$ R. M. Corn and M. R. Philpott, J. Chem. Phys. 80, 5245 (1984).

${ }^{51}$ D. G. Lidzey, A. I. Tartakovskii, M. Emam-Ismail, M. S. Skolnick, and S. Walker, Synth. Met. 127, 151 (2002).

${ }^{52}$ Y. Mori, J. Raman Spectrosc. 32, 543 (2001). 